\title{
The role of Tks adaptor proteins in invadopodia formation, growth and metastasis of melanoma
}

\author{
Shinji Iizuka1,2, Christopher Abdullah"1,2,3, Matthew D. Buschman ${ }^{1,4}$, Begoña Diaz ${ }^{1,5}$ \\ and Sara A. Courtneidge $\mathbf{e}^{1,2,6}$ \\ ${ }^{1}$ Sanford|Burnham|Prebys Medical Discovery Institute, La Jolla, CA, USA \\ ${ }^{2}$ Department of Cell, Developmental \& Cancer Biology, Oregon Health \& Science University, Portland, OR, USA \\ ${ }^{3}$ Biomedical Sciences Graduate Program, University of California, San Diego, La Jolla, CA, USA \\ ${ }^{4}$ Department of Medicine, Division of Endocrinology and Metabolism, University of California, San Diego, La Jolla, CA, USA \\ ${ }^{5}$ Los Angeles Biomedical Research Institute at Harbor-UCLA Medical Center, Torrance, CA, USA \\ ${ }^{6}$ Department of Biomedical Engineering and Knight Cancer Institute, Oregon Health \& Science University, Portland, OR, USA \\ Correspondence to: Sara A. Courtneidge, email: courtneidge@ohsu.edu
}

Keywords: invadopodia, Tks5, Tks4, MT1-MMP, melanoma

Received: August 11, $2016 \quad$ Accepted: October 22, $2016 \quad$ Published: October 27, 2016

\section{ABSTRACT}

Metastatic cancer cells are characterized by their ability to degrade and invade through extracellular matrix. We previously showed that the Tks adaptor proteins, Tks4 and Tks5, are required for invadopodia formation and/or function in Srctransformed fibroblasts and a number of human cancer cell types. In this study, we investigated the role of Tks adaptor proteins in melanoma cell invasion and metastasis. Knockdown of either Tks 4 or Tks5 in both mouse and human melanoma cell lines resulted in a decreased ability to form invadopodia and degrade extracellular matrix. In addition, Tks-knockdown melanoma cells had decreased proliferation in a 3-dimensional type I collagen matrix, but not in 2-dimensional culture conditions. We also investigated the role of Tks proteins in melanoma progression in vivo using xenografts and experimental metastasis assays. Consistent with our in vitro results, reduction of Tks proteins markedly reduced subcutaneous melanoma growth as well as metastatic growth in the lung. We explored the clinical relevance of Tks protein expression in human melanoma specimens using a tissue microarray. Compared to non-malignant nevi, both Tks proteins were highly expressed in melanoma tissues. Moreover, metastatic melanoma cases showed higher expression of Tks5 than primary melanoma cases. Taken together, these findings suggest the importance of Tks adaptor proteins in melanoma growth and metastasis in vivo, likely via functional invadopodia formation.

\section{INTRODUCTION}

Tumor cell metastasis is a complex, multistep process, in which tumor cells escape from the primary tumor and form secondary tumors at distant sites. Several steps involved in the metastatic cascade, including tumor dissemination, intra- and extravasation, as well as colonization and tumor growth at distant organs, require the cells to gain the ability to degrade and remodel the surrounding extracellular matrix (ECM) $[1,2]$.

Invadopodia have been described as one of the key regulators of cancer metastasis [3-5]. Invadopodia are actin-rich cell membrane protrusions that extend from the ventral surface of invasive cells in in vitro twodimensional (2D) culture and display focal proteolytic activity towards the ECM $[6,7]$. These cellular projections were first discovered in Src-transformed fibroblasts, where they were initially called podosomes [8], and were subsequently identified in a variety of invasive human cancer cells, where the term invadopodia was coined [9, 10]. Invadopodia are important regulators of proteasedependent cell invasion [11, 12].

A key regulator of invadopodia formation, the adaptor protein Tks5 (tyrosine kinase substrate with five 
SH3 domains - previously known as Fish), was originally discovered in our laboratory as a Src substrate [13-15]. Tks 5 is encoded by the $S H 3 P X D 2 A$ gene and contains a Phox-homology (PX) domain located at the N-terminus, five SH3 domains, as well as several polyproline motifs and two Src phosphorylation sites [16]. Tks5 plays a role in the function and formation of both podosomes and invadopodia [14, 16-20]. Our laboratory has demonstrated that Tks5 is required for mammalian development and cancer progression $[6,14,19,21-24]$. We have also described the Tks4 (tyrosine kinase substrate with four SH3 domains) protein, a close homolog of Tks5, as a critical invadopodia component in Src-transformed fibroblasts [25], as well as a regulator of developmental processes [22, 26, 27]. Loss of Tks4 in Src-transformed fibroblasts resulted in the formation of pre-invadopodia structures, where many of the required structural and accessory proteins were appropriately localized, but ECM degradation did not take place [25]. However, Tks4 has not been studied in human cancer.

Cysteine, serine and metalloproteases (MMPs) are all found at invadopodia [6, 7]. Of all MMPs, MT1MMP (also known as MMP14) appears to have the most significant role in cancer cell migration and invasion into the ECM [28, 29], likely through its diversity of substrates. MT1-MMP proteolytically activates other MMPs, such as MMP-2 and -13. It also directly cleaves many ECM components including type-I, -II and -III collagens, gelatin, fibronectin, fibrin, laminins 1 and 5, and vitronectin [30]. Regulation of MT1-MMP activity is thus a critical component of the invasive capacity of a cell. In particular, the subcellular localization of MT1-MMP plays an important role in regulating its function. MT1MMP localization is controlled by its transmembrane domain and its 20 amino acid-long cytoplasmic tail. The cytoplasmic tail is critical for correct MT1-MMP localization and activity [31-33]. Once internalized, MT1MMP can either be targeted for degradation or recycled back to the cell membrane $[34,35]$. Thus, the surface expression and targeting of MT1-MMP to specific areas of the cell surface, particularly at invadopodia, represents a key mechanism for regulating its proteolytic activity. However, the regulation of cell surface targeting is incompletely understood [36].

Interestingly, there is also evidence that overexpressed MT1-MMP can promote growth in threedimensional (3D) ECM [37], raising the possibility that the proteolytic properties of invadopodia might also be involved cancer cell growth. In keeping with this, our previous studies have suggested a role for Tks 5 in growth in a more physiological 3D ECM context as well as in vivo $[19,21]$, in contrast to our previous findings that Tks5 was not required for cancer cell growth, which were based on studies performed in monolayer cell culture [14].

Our recent work has suggested the importance of Tks5 in cancer progression in vivo using a breast cancer orthotopic graft model [21]. Additionally, studies have demonstrated the clinical relevance of Tks5 expression in cancer, such as glial-derived brain tumors, lung adenocarcinomas, prostate cancer and breast cancer [21, 38-40]. However, the requirements for Tks adaptor proteins in melanoma are not well studied. Here, we explore the role of Tks4 and Tks5 in melanoma growth and metastasis and the clinical relevance of these proteins in human melanoma patient samples.

\section{RESULTS}

\section{Tks adaptor proteins are required for functional invadopodia formation and mouse melanoma growth}

Stylli et. al. have previously shown that invadopodia formation plays a role in mouse melanoma invasion via the Src-Tks5 pathway in cells overexpressing constitutively active Src and/or Tks5 [16]. However, the role of endogenous Tks5 in melanoma growth has not been studied. Moreover, the role of Tks4 in melanoma progression is not known. To investigate the roles of Tks adaptor proteins in melanoma, we began by using B16F10 cells (a highly metastatic murine melanoma cell line derived from spontaneously arising melanoma in C57BL/6 mice [41]) to generate cells that stably expressed shRNA specific for Tks4, Tks5 or a scrambled control sequence. Efficient knockdown of Tks4 or Tks5 was achieved (Supplementary Figure 1). In comparison to the scrambled shRNA-expressing control cells $(72 \%$ invadopodia-positive), knockdown of either Tks4 or Tks5 in B16F10 cells resulted in a decrease in their ability to form invadopodia on Matrigel-coated glass coverslips $(6 \%$ and $2 \%$ invadopodia-positive cells respectively), as judged by co-staining with actin and cortactin (Figure 1A, 1B). Consistent with this result, knockdown of each of the Tks adaptor proteins also decreased the degradation activity on a gelatin matrix (Figure 1A, 1C). To complement these experiments, we also over-expressed GFP-tagged forms of Tks4 or Tks 5 into the B16F10 cells. In both cases, we observed co-localization of these scaffold proteins with cortactin (Figure 1D), as well as increased invadopodia formation when the cells were cultured on glass coverslips without added matrigel (Figure 1D, 1E). These data indicate that the expression level of Tks adaptor proteins modulate invadopodia formation in mouse melanoma cells.

It has previously been reported that cell proliferation in a 3D native (non-pepsin-extracted) type 1 collagen matrix required active MT1-MMP (in the case of cells engineered to overexpress the protease) and Tks5 (in the case of the endogenous protein in breast cancer cells) $[21,37]$. We evaluated the requirement for Tks4 and 
Tks5 in mouse melanoma cells using this 3D collagen assay (Figure 1F). In comparison to scrambled control cells, knockdown of Tks4 or Tks5 in B16F10 cells resulted in an approximately 40\% decrease (after 9 days in culture) in cell proliferation when embedded in a type 1 collagen matrix, but not when the cells were grown in $2 \mathrm{D}$ conditions (both on plastic dishes and on top of type 1 collagen) (Figure 1F). These data suggest that Tks adaptor proteins are also required for optimal 3D melanoma growth.

\section{Knockdown of Tks4 or Tks5 decreases the ability of B16F10 melanoma cells to colonize the lungs}

The use of the B16F10 cells allowed us to test whether Tks4 and Tks5 play a role in vivo, in immunocompetent animals. The scrambled and Tks adaptor protein knockdown cells were injected into the circulation through the tail vein, and the lungs were subsequently examined for tumor colonization, a so-called experimental metastasis assay. We also tried to examine tumor growth using a xenograft model; however, these data were difficult to analyze accurately because the highly
A
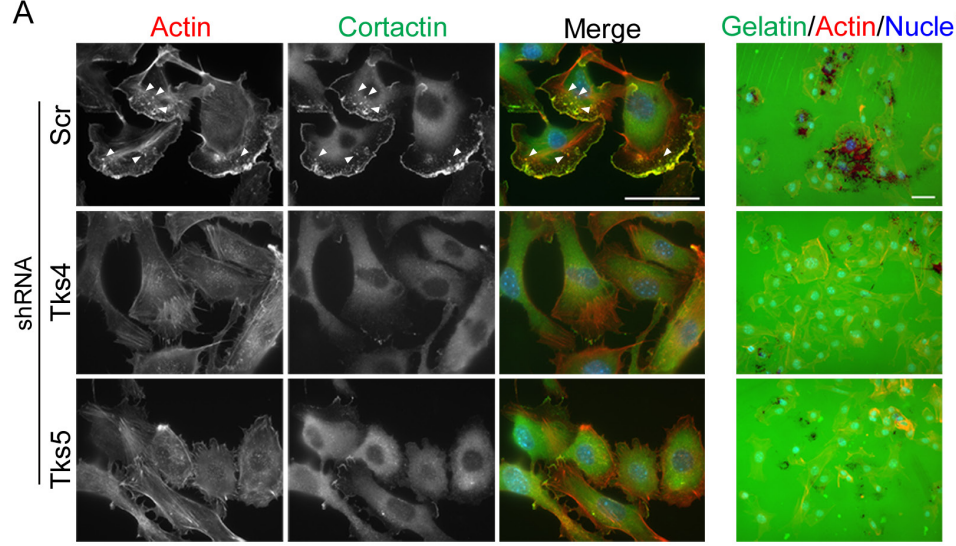

$\mathrm{D}$

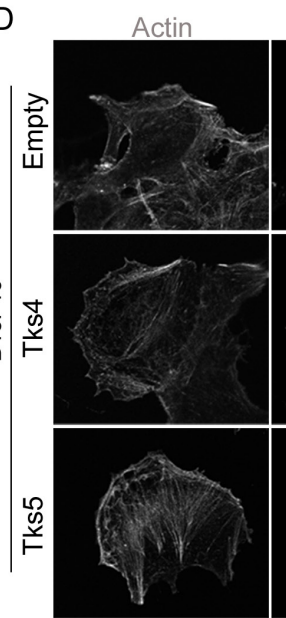

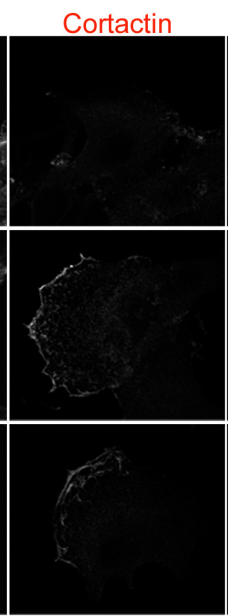

GFP
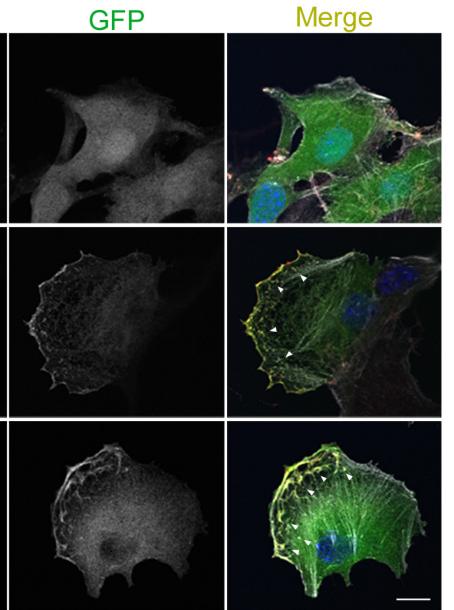

B

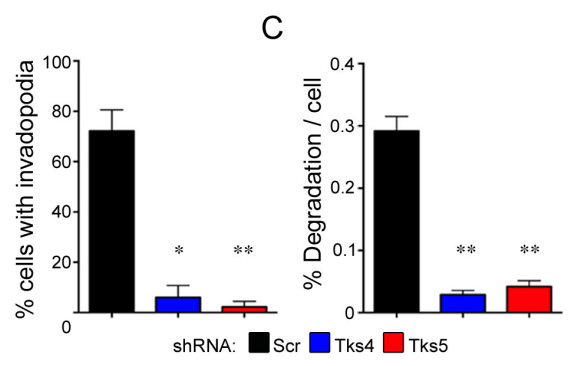

$\mathrm{E}$

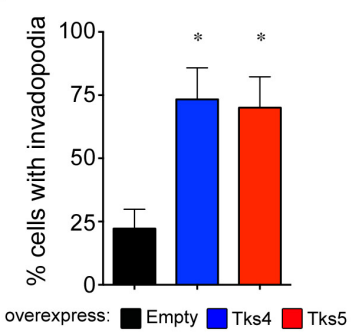

F

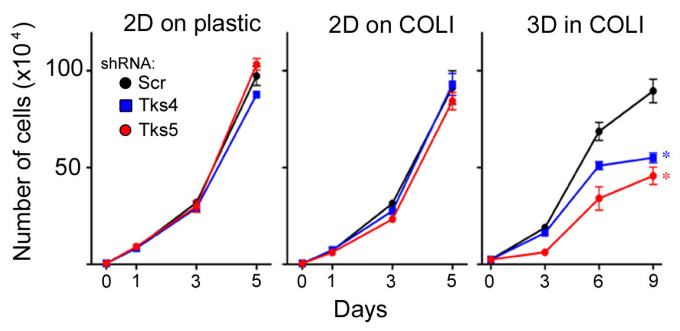

Figure 1: Invadopodia formation and function are required for mouse melanoma cell invasion and growth in 3D culture. A. B16F10 cells with Scr, Tks4 or Tks5 knockdown were analyzed for invadopodia formation (left, grown on matrigel-coated coverslips) and ECM-degradation activity (right, grown on gelatin-coated coverslips). Cells were stained with F-actin (red) and cortactin (green) for invadopodia detection, and with Hoechst for detection of nuclei (blue). Representative invadopodia are highlighted with white arrows. Bars: $50 \mu \mathrm{m}$. B.,C. Quantification of invadopodia formation and function. Percent of invadopodia-positive cells and gelatin degradation activity in B16F10 cells with Scr, Tks4 or Tks5 knockdown. ${ }^{*} p<5 \times 10^{-4}$; ${ }^{*} p<10^{-4}$. D. B16F10 cells transfected with empty GFP (empty), Tks4-GFP (Tks4) or Tks5-GFP (Tks5) were analyzed for invadopodia formation. Invadopodia were visualized by F-actin (gray) and cortactin (red) on glass coverslips without matrigel coating). Nuclei were stained with Hoechst (blue). Representative invadopodia are highlighted with white arrowheads. Bars: $10 \mu \mathrm{m}$. E. Quantification of invadopodia formation. Percent of invadopodiapositive cells was assessed. Data are presented as means \pm SEM. ${ }^{*} p<0.05$. F. Growth of B16F10 cells with Scr, Tks4 or Tks5 knockdown were analyzed in 2D conditions (plastic dish and type 1 collagen; 2D on COLl) and in 3D condition (3D in COLl). ${ }^{*} p<0.05, * * p<0.01$. Data are presented as means \pm SEM; for B. $n=9$ of each graph; for $\mathbf{F}$. $n=3$. Statistical analysis for the growth curves was performed by comparing the AUC for each condition (Supplementary Figure 7). 
invasive B16F10 cells disseminated from the primary site, and the resulting tumors could not be accurately measured or dissected from mice (not shown). Mice injected in the tail vein with the scrambled shRNA expressing melanoma cells showed considerable colonization of the lungs (Figure 2A), however there was a significant reduction in both lung tumor number and size in the Tks4 and Tks5 knockdown cells (Figure 2A, 2B). Taken together, these results suggest that Tks4 and Tks5 are important regulators of mouse melanoma growth in vivo.

\section{Tks adaptor proteins expression in human melanoma tissues}

To investigate the potential clinical relevance of Tks4 and Tks5 expression in human melanoma, we performed immunohistochemical (IHC) analysis of tissue microarrays containing human melanoma specimens (total of 110 cases for Tks4 and 165 cases for Tks5 analysis). We used commercially available rabbit polyclonal Tks5 and Tks4 antibodies, which we had previously validated [21]. Each stained sample was blindly scored by a trained pathologist (SI) and representative images are shown in Figure 3A and 3B (top panels). Tissue microarray specimens also included nevi as a non-malignant tissue control (12 cases for Tks4 and 50 cases for Tks5 analysis). Nevi were predominantly weakly positive $(1+)$, and there were no strong positives $(3+)$ for either Tks4 or Tks5 expression. Compared to nevi samples, expression of Tks4 and Tks5 were largely positive $(2+)$ or strongly positive $(3+)$ in melanoma specimens. Interestingly, specimens with strong positive staining of Tks 5 were highly increased in metastatic cases (lymph node metastasis: $52 \%$ and all other metastases: $72 \%$ ) compared to primary melanoma cases $(25 \%)$. A progression-dependent expression pattern was not found for Tks4, instead Tks4 expression was largely strong positive $(3+)$ in all melanoma specimens. These analyses suggest that both expression of Tks4 and Tks5 were increased in human melanoma when compared to nevi (Figure 3A and 3B, bottom panels). These expression patterns were statistically significant using chi-squared analysis $(p<0.05)$. It has recently been reported that there are multiple isoforms of Tks5, generated by distinct promoters, and known as $\alpha, \beta$ and short [22, 39], and that it is only expression of the $\alpha$ form which correlates with outcome for lung and breast cancer $[21,39]$. Currently available antibodies do not distinguish between the three isoforms. However, we could evaluate their expression in melanoma cell lines using isoformspecific qPCR and immunoblotting (supplementary Figure 2). We found that all but one cell line expressed Tks $5 \alpha$, at both the mRNA and protein level. The $\beta$ isoform was generally not expressed. The short isoform was detectable at the mRNA level in all but one cell line, although interestingly protein expression was always low.
A

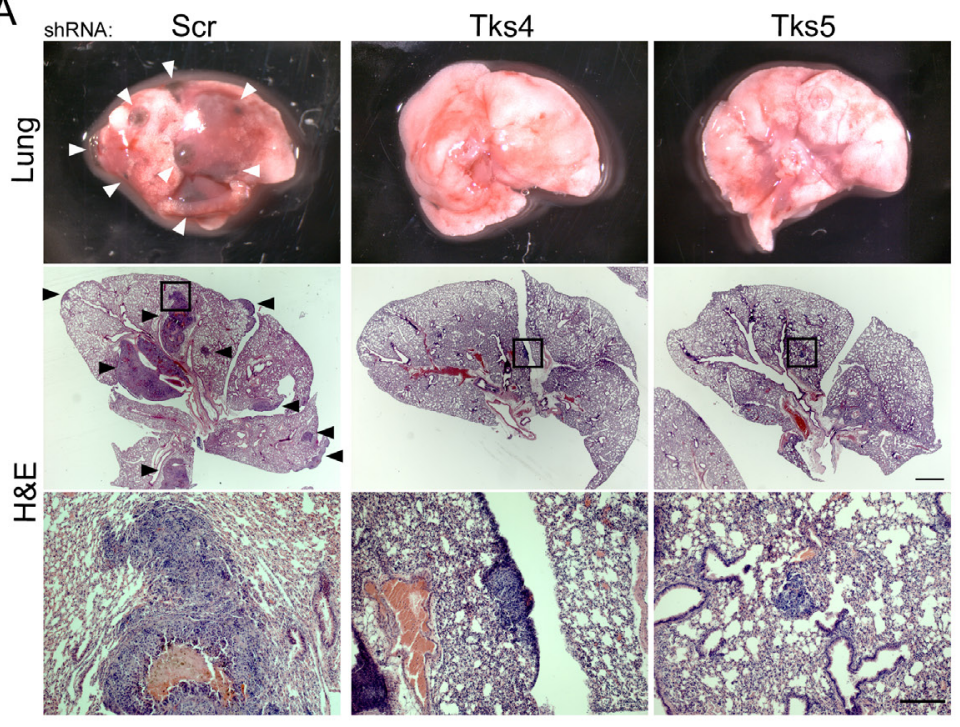

B

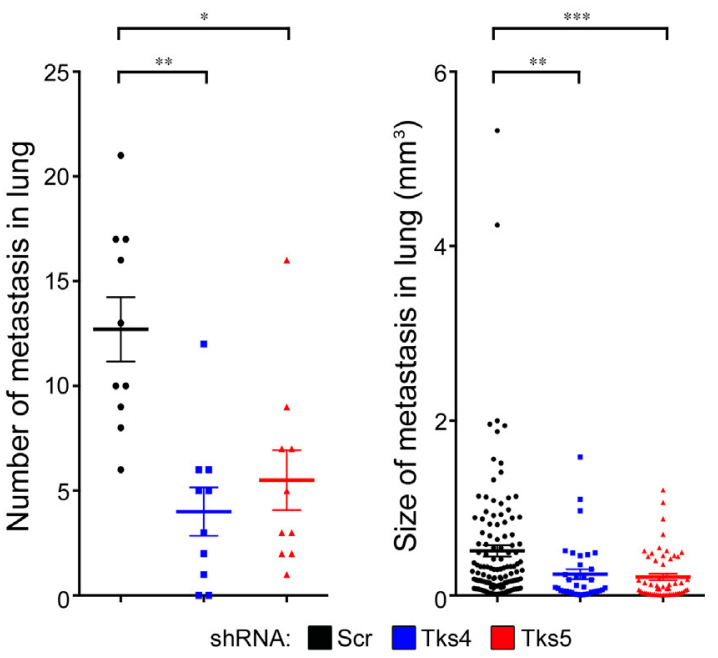

Figure 2: Tks adaptor proteins are required for melanoma metastasis in vivo. $\mathrm{C} 57 \mathrm{Bl} / 6$ mice were injected intravenously with $1 \times 10^{5}$ B16F10 cells with Scr, Tks4 or Tks5 knockdown. After 28 days, the number of metastases per lung and size of each lung metastasis were evaluated. A. Representative macroscopic views of lung (top panels) and H\&E-stained lung sections (middle and bottom panels). Arrowheads indicate metastatic melanoma in the lung, and high-magnification images (bottom panels) are shown from the indicated area in the middle panels. B. The number of lung metastases per lung and the size of each lung metastasis were determined by serial sections of each lung with H\&E-staining. ${ }^{*} p<0.005, * * p<0.001,{ }^{* * *} p<10^{-4}$. Data are presented as means $\pm \mathrm{SEM}$; for (B, left) $n=10$ for each group; for (B, right) $n=$ Scr: 122, Tks4: 36, Tks5: 57. Scale bar: $1 \mathrm{~mm}$ (middle panel), $200 \mu \mathrm{m}$ (lower panel). 


\section{Tks adaptor proteins are required for functional invadopodia formation and 3D growth in human melanoma cells}

We next wanted to examine the role of Tks adaptor proteins in human melanoma. We previously demonstrated that knockdown of Tks5 in the human melanoma cell lines, C8161.9 and RPMI-7951, decreased melanoma cell invasion in vitro [14], but we did not quantify invadopodia at that time. There are no published reports on the role of Tks4 in any human cancer, except for the analysis of invadopodia function in one colon cancer cell line engineered to express constitutively active $\mathrm{Src}$ [42]. We first examined Tks4 and Tks5 localization in the human melanoma cell line C8161.9, and determined their localization to invadopodia (supplementary Figure 3). Tks 4 or Tks5 were stably knocked down using two distinct shRNAs (shRNA-75 and -68 for Tks4, shRNA-D6 and D7 for Tks5) and then invadopodia formation and function evaluated in both C8161.9 (Figure 4) and WM793 (supplementary Figure 4). More than $70 \%$ of the cells formed invadopodia in control cells (88\% in C8161.9 cells and $74 \%$ in WM793 cells), but knockdown of Tks4 or Tks5 led to significant decreases in invadopodia formation (less than $30 \%$ in each case). Consistent with these results, knockdown of Tks 4 and Tks5 also significantly decreased the degradation of gelatin matrix. These data demonstrate that Tks adaptor proteins were required for functional invadopodia formation in human melanoma cells.

We next investigated the role of Tks proteins in human melanoma growth. Cells were placed in 3D matrices of native type 1 collagen, and growth compared to that in $2 \mathrm{D}$ (on plastic or on type 1 collagen) using both C8161.9 and WM793 cells with knockdown of Tks5 (D6 or D7) and Tks4 (75 and 68). Every 2-4 days, the total number of cells was counted, and the growth rates assessed (Figure 4E and Supplementary Figure 4). C8161.9 and WM793 cells with Tks4 or Tks5 knockdown proliferated normally under 2D culture conditions compared to scrambled controls (on plastic and on type 1 collagen). However, when these cell lines were cultured in 3D type 1 collagen, growth rates of Tks4- or Tks5-knockdown cells were significantly decreased compared to scrambled control cells. Interestingly, another invadopodia protein MT1-MMP, was also required for 3D, but not 2D growth of C8161.9 cells (supplementary Figure 5). These data indicate that Tks adaptor proteins, and likely functional invadopodia, were required for human melanoma growth in $3 \mathrm{D}$ conditions in vitro.

\section{Tks adaptor proteins are required for human melanoma progression in vivo}

We next conducted xenograft tumor growth assays and experimental metastasis assays in vivo (Figure 5). We first tested the appropriateness of C8161.9 and WM793 cell lines for in vivo experiments. C8161.9 and WM793 cells were subcutaneously injected into nude mice, and the size of tumor was measured every 2-3 days. In addition, these cell lines were also introduced via the tail vein to test metastatic ability. We noted that C8161.9 cells grew well in vivo both in the primary site and in the lung, but no
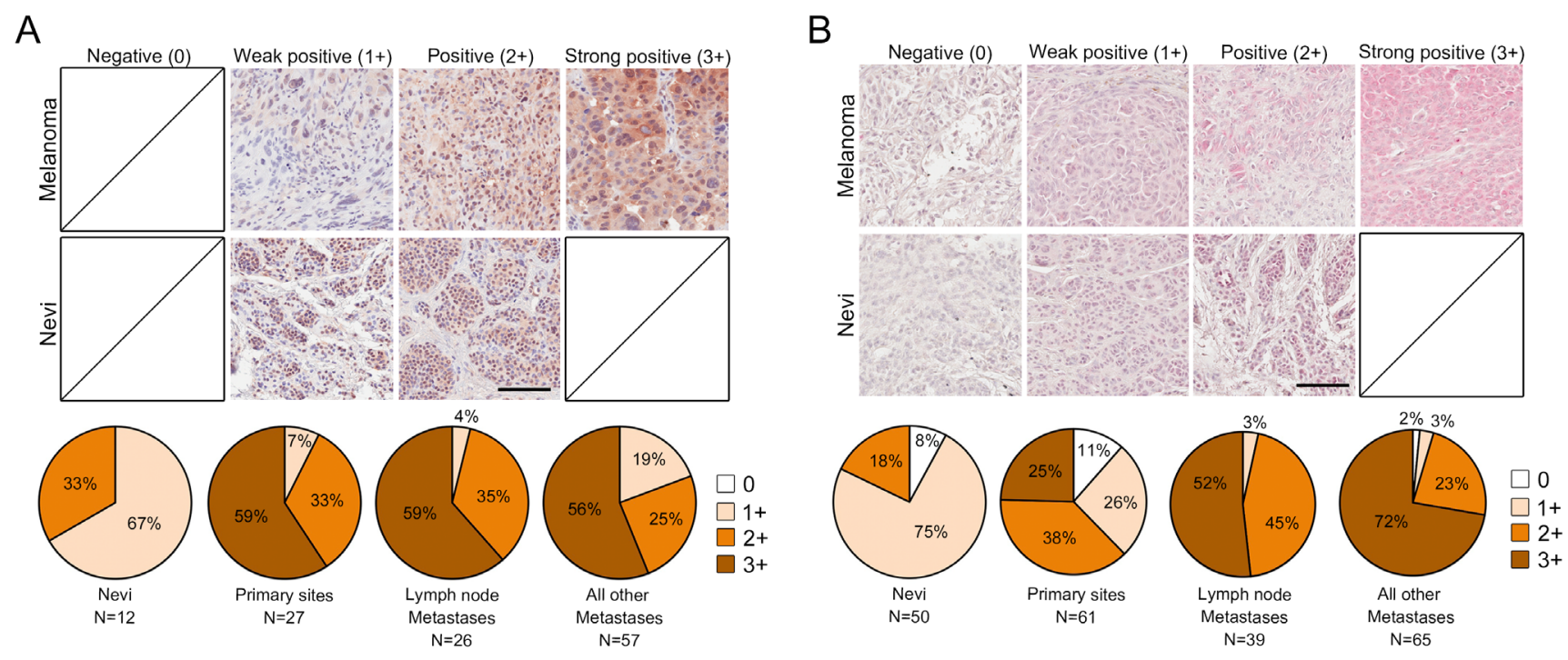

Figure 3: Tks adaptor proteins are highly expressed in human melanoma. Tks4 A. and Tks5 B. IHC staining of human melanoma tissue microarray ( $n=165$ for Tks5, $n=110$ for Tks4) including nevi as non-malignant control tissues $(n=50$ for Tks5, $n=12$ for Tks4). Representative images from melanoma cases and nevi are shown (top and middle panel). Scale bar $200 \mu \mathrm{m}$. The staining intensity was scored as four levels as indicated in top panels. Distribution of IHC staining intensity for Tks4 and Tks5 in each melanoma stage is shown in the bottom pie graphs. Chi-squared analysis was performed to test statistical significance between progression and staining intensity $(p<0.05)$. 
tumor growth at either primary site or lungs was observed with WM793 cells (data not shown). Therefore we used C8161.9 cells to test the role of Tks adaptor proteins in vivo. Melanoma cells with knockdown of either Tks4 or Tks5 showed significant reduction of primary tumor size compared to controls (panels A, B). Consistent with our previous results using mouse melanoma cells, Tks knockdown in human melanoma cells also led to significant decrease in both the number and the size of metastases in lungs after tail vein injection (panels C-E). Taken together, these results indicate that both Tks4 and Tks5 are required for human melanoma growth and experimental metastasis.

\section{Tks adaptor proteins regulate MT1-MMP cell surface expression}

MT1-MMP is known to be recruited to invadopodia and contributes to matrix degradation [28, 43, 44]. And our laboratory has previously described that Tks4 is required for MT1-MMP localization to invadopodia in Src-transformed fibroblasts [25]. In keeping with this, we found that MT1-MMP co-localized with GFPtagged Tks4, and to a lesser extent Tks5, in the human melanoma cell line C8161.9 (supplementary Figure 6). To assess whether Tks adaptor proteins regulate the cell surface expression of MT1-MMP in melanoma cells, we used two experimental approaches. First, the expression level of MT1-MMP at the cell surface was examined by

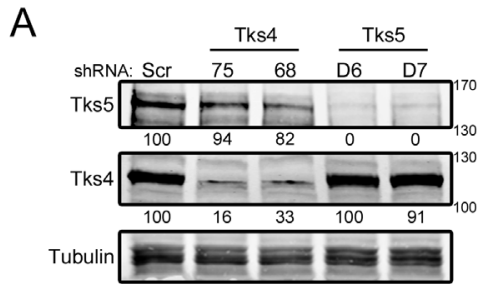

B

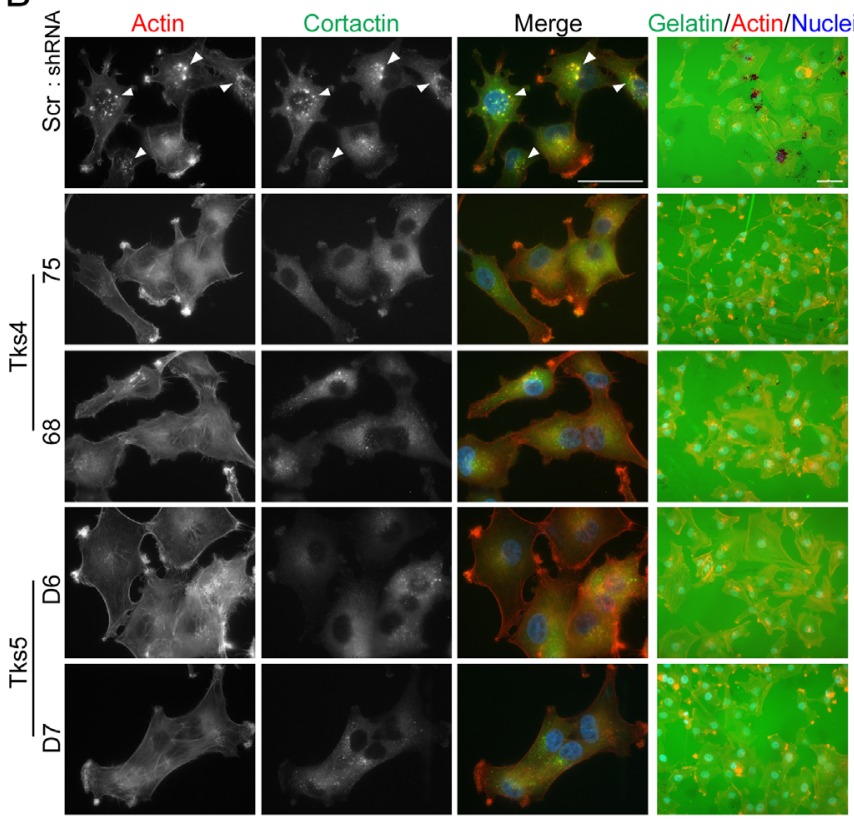

C

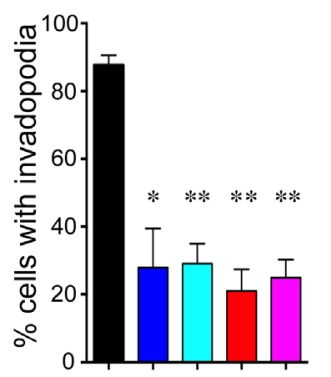

D

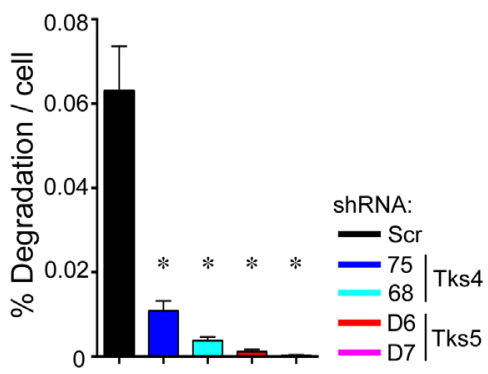

E

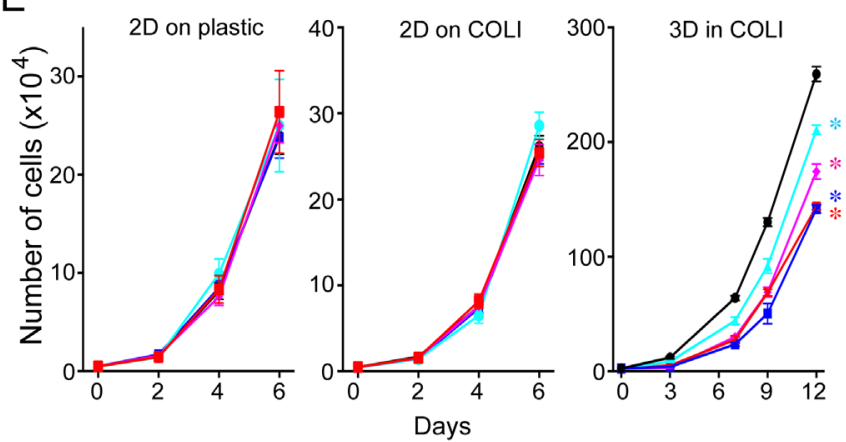

Figure 4: Tks adaptors are required for human melanoma invasion and growth in 3D culture conditions. The human melanoma cell line, C8161.9 was infected with scrambled (Scr), Tks4- or Tks5-specific shRNA lentiviruses and analyzed in invadopodia formation and function assays. A. Lysates from C8161.9 cells infected with indicated shRNA viruses were immunoblotted with each antibody in the figure. B. C8161.9 cells with Scr, Tks4 or Tks5 knockdown were analyzed for invadopodia formation (left) and ECMdegradation activity (right, grown on gelatin coat coverslips). Invadopodia were visualized by F-actin (red) and cortactin (green). Nuclei were stained by Hoechst (blue). Representative invadopodia are highlighted with white arrowheads. Bars: $50 \mu \mathrm{m}$. C., D. Quantification of invadopodia formation and function. Percent of invadopodia-positive cells and gelatin degradation activity in C8161.9 cells with Scr, Tks4 or Tks5 knockdown. E. Growth of human melanoma cell lines, C8161.9 with Scr, Tks4 (75 and 68) or Tks5 (D6 and D7) knockdown were analyzed on 2D conditions (plastic dish and type 1 collagen; 2D on COLl) and on 3D condition (3D in COLl). p-value for C. $* p<0.005$, ${ }^{* *} p<10^{-4}(n=9)$; for D. ${ }^{*} p<0.01(n=5)$. Statistical analyses were performed using Student's t test for C. and D.. Statistical analysis for the growth curves was performed by comparing the AUC for each condition (Supplementary Figure 7). 
a cell surface biotinylation pulldown assay (Figure 6A). Although knockdown of Tks proteins had no effect on the total cellular levels of MT1-MMP, cell surface expression of MT1-MMP was reduced in Tks protein knockdown cells (49\% reduction in Tks4 knockdown, $73 \%$ reduction in Tks5 knockdown). Second, these findings were confirmed by analyzing MT1-MMP exocytic events using pHluorin tagged MT1-MMP. We also overexpressed Lifeact-mCherry to visualize actin structures in these cells. The pHluorin-tag is a $\mathrm{pH}$-dependent green fluorescent protein (GFP) variant that is fluorescent at neutral $\mathrm{pH}$ (extracellular region), but not at acidic $\mathrm{pH}$ (endosomal vesicle) [45], and has been used to study MT1-MMP trafficking [46]. For these experiments, pHluorin-MT1MMP and Lifeact-mCherry overexpressing C8161.9 cells were infected with scrambled, shRNA-Tks4 and shRNATks5. The actin structures visualized by Lifeact-mCherry were used for focusing on the ventral surface of the cell, and the GFP flash at the cell surface was analyzed by timelapse confocal microscopy to visualize exocytic events (cell surface expression) of MT1-MMP (Figure 6B-6D). Consistent with our findings in the pulldown assay (Figure 6A), MT1-MMP exocytic events in the Tks4 and Tks5 knockdown cells were significantly reduced compared to control cells suggesting that both Tks4 and Tks5 are necessary for MT1-MMP surface expression, likely by regulating its exocytosis.

\section{DISCUSSION}

Previous findings have indicated that the adaptor protein Tks5 regulates melanoma invasiveness in vitro $[14,16]$. However, the role of Tks4 in cancer cells has not previously been addressed. In the present study, we demonstrated that both Tks4 and Tks5 were required for functional invadopodia formation, in both mouse and human melanoma cells. We previously reported that Tks4 is localized at invadopodia in Src-transformed fibroblasts, and that knockdown of Tks4 led to incomplete invadopodia formation as defined by reduced polymerized actin, lack of ECM degradation, and failure to accumulate MT1-MMP [25]. Overexpression of Tks5 rescued the actin polymerization at invadopodia, but not ECM degradation or MT1-MMP targeting [25]. We show here that in melanoma cells, both Tks4 and Tks5 were required for invadopodia formation and function, suggesting that they have non-overlapping functions in this cell type. Alternatively, it is also possible that Tks4 and Tks5 have similar functions, and that the total dosage of these adaptor proteins is important. In this scenario, knockdown of either Tks 4 or Tks5 would reduce the level of the adaptors below a critical threshold required for invadopodia formation. In keeping with this latter hypothesis, Tks4 knockout MEFs started to re-form functional invadopodia structures via up-regulation of Tks5 after prolonged passage in culture,
A

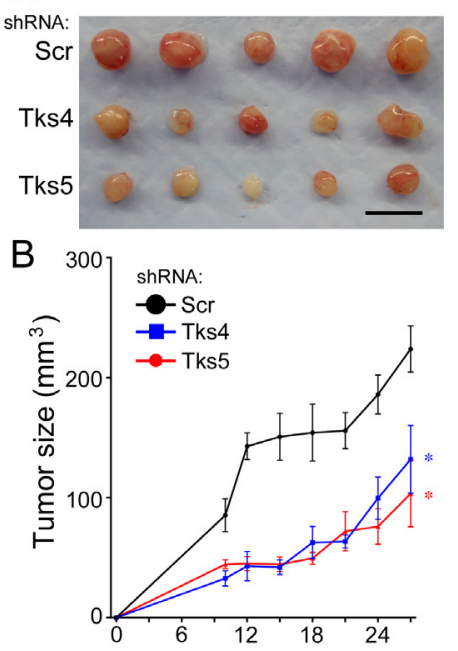

C

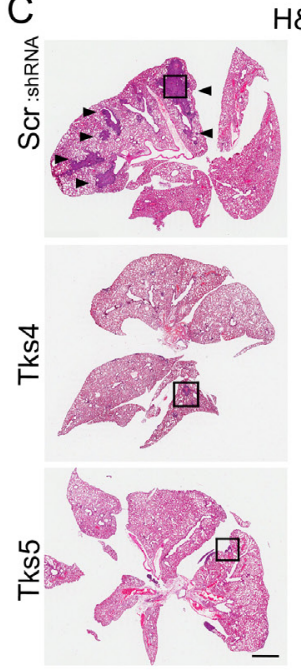

H\&E

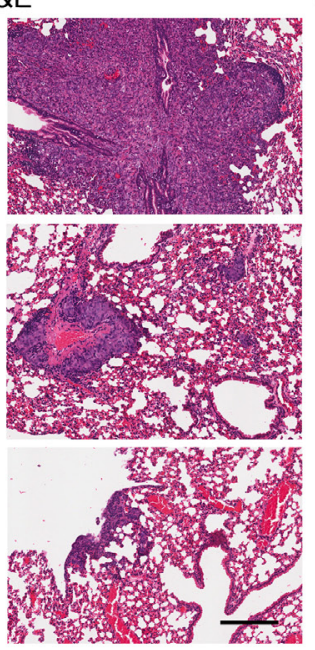

D

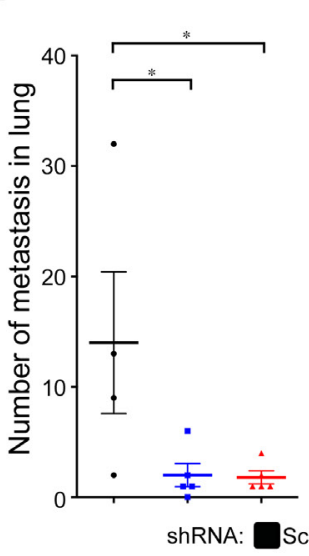

$\mathrm{E}$

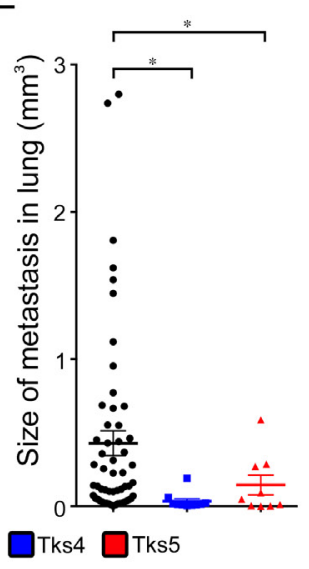

Figure 5: Tks adaptor proteins are required for human melanoma growth and metastasis in vivo. For the tumor growth assay, nude mice were subcutaneously injected with 4 x $10^{5}$ C8161.9 cells with Scr, Tks4 (75) and Tks5 (D6) knockdown. A. Macroscopic view of all tumors ( $n=5$ in each group) is shown. Scale bar: $10 \mathrm{~mm}$. B. Tumor volumes were measured every 2-3 days until 27 days after injection. $* p<0.05, * * p<0.01, * * * p<0.005, * * * p<5 \times 10^{-4}$. Statistical analysis for the growth curves was performed by comparing the AUC for each condition (Supplementary Figure 7). For the metastasis assay, nude mice were intravenously injected with $1 \times 10^{5} \mathrm{C} 8161.9$ cells with Scr, Tks4 (75) and Tks5 (D6) knockdown. After 28 days, the lung metastases were evaluated. C. Representative H\&E-stained lung sections are shown. Arrowheads indicate metastatic melanoma in the lung and high-magnification images (right panels) are shown from the indicated area in left panels. Scale bar: $1 \mathrm{~mm}$ (left panel), $200 \mathrm{~mm}$ (right panel). The number of lung metastases per lung D. and size of each lung metastasis E. were evaluated by serial sections of each lung with H\&E-staining. One of the mice in the control group (scrambled shRNA) died during the experiments. p-values for D. ${ }^{*} p<0.05$; for $\mathbf{E} .{ }^{*} p<0.05$. Data are presented as means \pm SEM; for B. $n$ $=5$ for each group; for D. $n=$ Scr: 4, Tks4 and Tks5: 5; for E. $n=$ Scr: 56, Tks4: 10, Tks5: 9. 
or upon overexpression of Tks5 [25]. Furthermore, it has not proven possible to simultaneously knock down both Tks4 and Tks5 (unpublished). Further studies into the binding partners and regulation of the Tks proteins are required to fully understand their distinct and possibly overlapping roles.

What is the mechanism by which the Tks adaptors, and invadopodia, regulate invasive capacity? It is known that MT1-MMP is one of the key proteases required for degradation of the ECM surrounding cancer tissue [47], and that MT1-MMP localizes to invadopodia [48]. The trafficking of MT1-MMP is a highly regulated process [28], involving, for example, an exocytic mechanism connecting MT1-MMP positive late endosomes and the plasma membrane, which requires ARF6 and JIP3/4 [49, 50]. We show here that reduced exocytosis of MT1-MMP vesicles following Tks adaptor knockdown accounted for the loss of surface expression of MT1-MMP. It is possible that loss of invadopodia caused a concomitant loss of exocyst docking sites, although future studies will be necessary to understand fully the mechanism.

High expression of Tks5 has previously been reported in some cancer types, such as glial-derived brain tumors [38], lung adenocarcinomas [39], prostate cancer [40] and breast cancer [21], and can correlate with poorer outcome $[21,38,39]$. Here, we show that high expression of Tks5, as well as Tks4, was also present in human melanoma samples. Moreover, higher expression levels of Tks 5 were more frequent in metastatic melanoma cases, compared to primary tumors, although this was not observed for Tks4. These findings prompted us to evaluate the roles of these adaptor proteins in melanoma progression and metastasis. Using an experimental metastasis assay, and both a mouse and a human melanoma cell line, we found fewer tumors in the lungs of mice injected with Tks4 or Tks5 knockdown cells, compared to the scrambled controls. This is consistent with a recent report that the Tks adaptors, and invadopodia, are required for extravasation in experimental model systems [4]. However, we also noticed that those metastases that did form in the lungs of mice injected with knockdown cells were smaller than controls, suggesting that Tks adaptors were also required for efficient tumor growth. In keeping with this, C8161.9 also grew more poorly as subcutaneous tumors in the absence of Tks4 or Tks5. In previous studies we noted reduced growth of Src-transformed fibroblasts and human breast cancer cells in subcutaneous and orthotopic sites respectively, when Tks5 expression

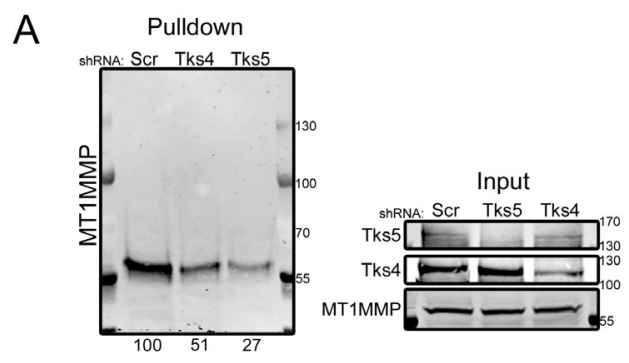

B

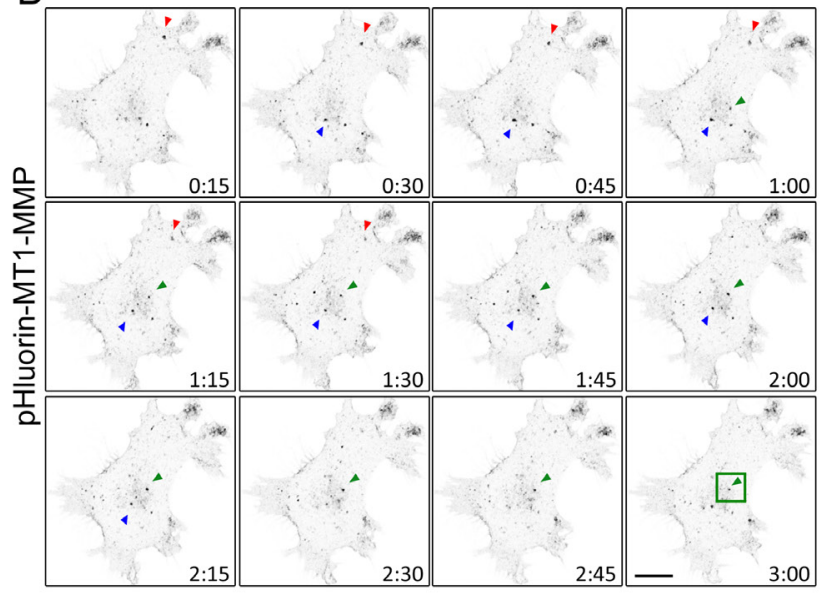

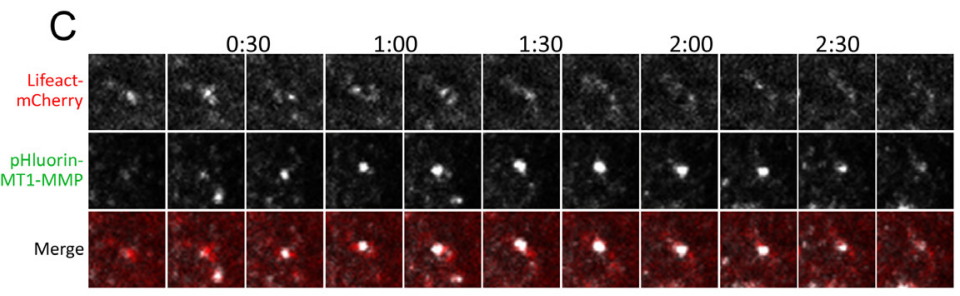

D

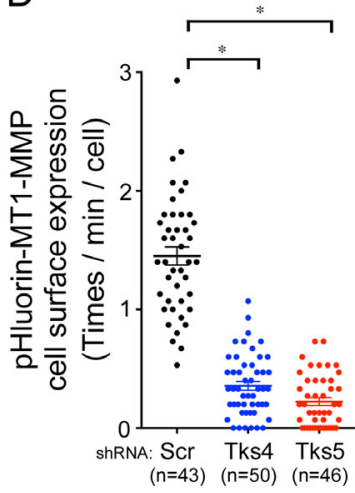

Figure 6: Tks adaptor proteins regulate surface expression of MT1-MMP. A. Cell surface expression of MT1-MMP in C8161.9 cells with Scr, Tks4 or Tks5 knockdown. Cells were labeled with biotin, and cell surface proteins were pulled down, using streptavidin-agarose beads and blotted by MT1-MMP antibody. Total expression levels of each protein were shown as loading controls. B. Time-lapse images (15 seconds per frame) of pHluorin expression at cell surface in C8161.9 with scrambled shRNA. Three representative GFP flashes were indicated by arrowheads with different color (Red, Green and Blue). C. High magnification images with Lifeact-mCherry (Red) and pHluorin-MT1-MMP (Green) from the green square shown in B.. D. Quantification of exocytic event (cell surface expression of MT1-MMP). n, total number of cells analyzed for each stable cell line. Data are presented as means \pm SEM. 
levels were reduced [19, 21]. Importantly, our use of the B16F10 cells introduced syngeneically into C57B16 mice has allowed us to rule out the possibility that these findings only apply to immunocompromised mice. It has previously been shown that tyrosine phosphorylation of Tks5 is required for invadopodia formation in melanoma cells [16], however whether this phosphorylation is catalyzed by a Src family kinase is as yet unexplored.

We have also shown both here and in previous work using a breast cancer cell line [21] that decreased levels of Tks adaptor proteins resulted in reduced growth in 3D-ECM culture conditions. It is formally possible that Tks4 and Tks5 have other functions, separate from their roles in invadopodia formation, which are necessary for tumor cell growth in 3D in vitro and in vivo. However, we note that other invadopodia proteins, for example MT1MMP (supplementary Figure 5 and [37]), Cdk5 [51] and cortactin [52], are also necessary for 3D and/or in vivo growth in pancreatic cancer and head-and-neck squamous cell cancers. Collectively, these data suggest that the prior almost exclusive use of 2D culture systems has led us to overlook a role for invadopodia in cancer cell growth. How 3D growth might be facilitated by invadopodia is not yet clear, although proteolysis is certainly likely to be involved. One possibility is that the proteolytic activity of these structures is required to create physical space to grow. Alternatively, growth factor and cytokine processing by proteases, including of the MMP class [53], may be necessary to overcome the restrictions in place in $3 \mathrm{D}$ environments. We are currently investigating these possibilities.

In summary, our findings indicate that both Tks4 and Tks5 have essential roles for melanoma growth and metastasis in vivo. Our data suggest a model where, through the Tks adaptor proteins, invadopodia formation and surface recruitment of MT1-MMP drive tumor growth and metastasis. Moreover, Tks adaptor proteins are highly expressed in melanoma patient samples. Although the full molecular mechanism of invadopodia-mediated cancer progression remains to be elucidated, we speculate that invadopodia inhibitors might have potential as therapeutic agents to prevent cancer progression.

\section{MATERIALS AND METHODS}

\section{Cell lines}

The C8161.9 human melanoma cell line was purchased from ATCC. The WM793 human melanoma cell line was a gift from Dr. Gary G. Chiang (Abbvie, Chicago, IL). The B16F10 mouse melanoma cell was a gift from Dr. Peter Lock (La Trobe University, Melbourne, Australia). Cells were grown in DMEM (Mediatech, Manassas, VA, USA) containing 10\% fetal bovine serum
(FBS). FBS was obtained from HyClone. All experiments were performed in complete medium in the absence of antibiotics.

\section{Reagents and antibodies for immunoblotting and immunohistochemistry}

The antibody for Tks5 (anti-Tks5 1737) was generated by the Courtneidge laboratory and used for immunoblotting and immunofluorescence experiments (Lock et al., 1998) and Tks5 antibody (ProteinTech) was used for immunohistochemistry, as previously described (Blouw et al., 2015). The anti-Tks4 antibody (09-267) was previously described (Buschman et al., 2009) and is available from EMD Millipore (Temecula, CA, USA). The following commercial antibodies were used: anticortactin (4F10) antibody (EMD Millipore), anti- $\gamma$ tubulin antibody (Sigma-Aldrich, St. Louis, MO, USA), MT1-MMP antibody (Abcam or EMD Millipore). Alexa Fluor 568-conjugated phalloidin (1:500 in PBS containing $0.5 \%$ BSA and $0.1 \%$ Triton X-100; EMD Chemicals Inc., Darmstadt, Germany) was used for actin staining. For secondary antibodies, Alexa Fluor 680 goat anti-rabbit IgG (Invitrogen, Carlsbad, CA, USA) or IR800 (Rockland Immunochemicals, Gilbertsville, PA, USA) were used for immunoblotting, and Alexa Fluor-594-conjugated or -488-conjugated antibodies (Chemicon, Temecula, CA, USA) were used for immunohistochemistry. For gelatin degradation assays, Oregon green gelatin $(0.2 \mathrm{mg} / \mathrm{ml}$ in PBS containing $2 \%$ sucrose; Invitrogen) was used.

\section{Immunoblotting}

Cell lysates were prepared by washing cells twice with cold Tris-buffered saline (TBS) containing $100 \mu \mathrm{M}$ $\mathrm{Na}_{3} \mathrm{VO}_{4}$ and then lysing in $50 \mathrm{mM}$ Tris- $\mathrm{HCl}(\mathrm{pH} 7.5), 250$ $\mathrm{mM} \mathrm{NaCl}, 1 \%$ Triton X-100, $50 \mathrm{mM} \mathrm{NaF}, 100 \mu \mathrm{M} \mathrm{Na}_{3} \mathrm{VO}_{4}$ and $1 \mathrm{mM}$ EDTA lysis buffer containing a dissolved complete Mini protease inhibitor tab (Roche Diagnostics, Germany). Supernatant of cell lysates was assayed for total protein content using the BCA protein assay (Thermo Fisher Scientific, Rockford, IL), and $70 \mu \mathrm{g}$ of total protein per sample was separated in a $7.5 \%$ polyacrylamide gel (Invitrogen). Secondary antibodies were conjugated to Alexa Fluor 680 or IR800, and membranes were scanned using an infrared imaging system (Odyssey; LI-COR Biosciences, Lincoln, NE).

\section{Biotin labeling of Cells}

Biotin labeling of cell surface proteins was performed as previously described [35]. Briefly, cells were washed twice in ice-cold PBS and incubated in PBS for $10 \mathrm{~min}$ at $4^{\circ} \mathrm{C}$. Cells were then labeled with biotin by 
incubating cells at $4^{\circ} \mathrm{C}$ with $0.5 \mathrm{mg} / \mathrm{ml}$ NHS-SS-Biotin (Thermo Fisher Scientific) for $30 \mathrm{~min}$. Biotinylated cells were lysed in cell lysis buffer, and labeled cell surface proteins were precipitated with streptavidin-agarose beads (Thermo Fisher Scientific).

\section{MT1-MMP-pHluorin}

C8161.9 cells stably expressing MT1-MMPpHluorin and Lifeact-mCherry were infected with the indicated shRNAs (scrambled, Tks5 and Tks4), and stable pools of cells were established. The cells were plated on glass bottomed dishes (MatTek, MatTek Corporation) and incubated for $6 \mathrm{hr}$ before imaging. The cells were imaged on a microscope (Zeiss LSM 880 laser-scanning confocal microscope with AiryScan, 4 images/min, total $15 \mathrm{~min}$ ). The number of exocytic events of MT1-MMP-pHluorin was manually counted and measured per minute and per cell.

\section{Invadopodia staining and gelatin degradation assay}

Invadopodia staining and gelatin degradation assays were performed as previously described [54]. Briefly, cells were grown on glass coverslips with or without Matrigel-coating (invadopodia formation assay) or gelatin-coated coverslips (degradation assay) and fixed with 4\% paraformaldehyde/PBS (Electron Microscopy Sciences). For the invadopodia assay on Matrigel-coated coverslips, BD Matrigel Basement Membrane Matrix (BD Biosciences, Bedford, MA, USA) was prepared according to the manufacturer's instructions. Briefly, Matrigel was diluted by serum-free medium (1:10 dilution) on ice and added to coverslips for $1 \mathrm{hr}$ at RT. After rinsing with serum-free medium, B16F10 cells were cultured on the coverslips and experiments performed. After permeabilization with $0.1 \%$ Triton X-100/PBS for $15 \mathrm{~min}$, the cells are incubated with primary antibodies overnight at $4^{\circ} \mathrm{C}$. Cells were washed and incubated with Alexa Fluor-conjugated secondary antibodies and phalloidin. Fluorescence microscopy images were obtained with a fluorescent microscope (Axioplan2; Carl Zeiss) equipped with a charge-coupled device camera (AxioCam HRm; Carl Zeiss) and AxioVision software (Carl Zeiss). For each invadopodia experiment, the number of cells forming invadopodia was quantified in 5-9 microscope fields (63x) imaged randomly and percentage of invadopodia forming cells was assessed. For each ECM degradation experiment, 5-9 microscope fields (40x) imaged randomly. The percentage of degraded area was quantified with ImageJ software (National Institutes of Health) and normalized to the number of nuclei in that area was represented as " $\%$ degradation per cell".

\section{Constructs}

pLKO.1 shRNA lentiviral plasmids used for scrambled and human Tks5 knockdown were purchased from Sigma-Aldrich. The clones used were TRCN0000136014 (referred to as clone D6) and TRCN0000136512 (referred to as clone D7). Mission shRNA (pLKO.1) constructs against mouse and human Tks4, were obtained from Sigma-Aldrich. The clones used were TRCN0000147775 (referred to as clone \#75) and TRCN0000129568 (referred to as clone \#68). MT1MMP-pHluorin Lentivirus vector was kindly provided by Dr. Philippe Chavrier (Institut Curie, Paris). pEGFP-N1 DNA plasmids used for overexpression experiments were purchased from Clontech (Mountain View, CA). Human Tks4 or Tks5 was overexpressed using Lipofectamine 2000 from Invitrogen (Carlsbad, CA, USA) in either B16F10 or C8161.9 cells according to the manufacturer's protocol. Two days post-transfection, cells were visualized for invadopodia formation.

Lentiviral preparations were made by the viral core facilities at the Sanford|Burnham|Prebys Medical Discovery Institute (La Jolla, CA) and Oregon National Primate Research Center (Molecular \& Cell Biology, Lentivirus Service) at the Oregon Health and Science University (Portland, OR).

\section{D proliferation assay}

Type 1 collagen 3D cultures were performed as described previously [37]. Briefly, rat tail type 1 collagen (BD Biosciences, Bedford, MA, USA) was prepared to a final concentration of $2 \mathrm{mg} / \mathrm{ml}$, and 5,000 to 25,000 cells were added to the collagen mix before gelling. Spread cells were grown for 9-14 days in DMEM containing $10 \%$ FBS. The matrix was dissolved with $2 \mathrm{mg} / \mathrm{ml}$ collagenase (Worthington, NJ, USA) and cell numbers were determined by hemocytometry.

Type 1 collagen 2D cultures were performed according to the manufacturer's instructions. Briefly, rat tail type 1 collagen (BD Biosciences) was prepared at a final concentration of $50 \mu \mathrm{g} / \mathrm{ml}$ in $0.02 \mathrm{M}$ acetic acid and incubated with coverslips at room temperature for $1 \mathrm{~h}$. After aspirating the remaining solution, it was rinsed well with PBS to remove any remaining acid. 5,000 to 10,000 cells were added and cultured for 5-6 days in DMEM containing 10\% FBS, and cell numbers were counted. The proliferation assay on plastic plates was performed by the same method described previously without type 1 collagen. 
Table 1: Primer sets for human Tks5 isoforms and housekeeping gene

\begin{tabular}{|l|l|}
\hline hTks5 alpha-F: & TAATCAATGTGACCTGGTCTG \\
\hline hTks5 alpha-R: & TTGGGGTCCTTCTGGCCAC \\
\hline hTks5 beta-F: & TGTCTATCTGTTGTTGCTTCTTTTTC \\
\hline hTks5 beta-R: & CAGAAGTGCTCACGAACCAC \\
\hline hTks5 short-F: & TGGCTCACCGCGTGCTTTCTG \\
\hline hTks5 short-R: & CAGAAGTGCTCACGAACCAC (same as beta-R) \\
\hline hRPLP0-F: & GGCGACCTGGAAGTCCAACT \\
\hline hRPLP0-R: & CCATCAGCACCACAGCCTTC \\
\hline
\end{tabular}

\section{Subcutaneous tumor growth and experimental metastasis assays}

All animal experiments were conducted in accordance with the NIH Guide for the Care and Use of Laboratory Animals. Subcutaneous implantation was carried out with minor modifications as described previously [19]. In short, cells were harvested by trypsinization and resuspended in PBS/Matrigel mixture (1:1 ratio) to a final concentration of $4 \times 10^{6} \mathrm{cells} / \mathrm{ml}$. Athymic nude mice (Harlan, Indianapolis, Indiana) were injected in the flank with $100 \mu \mathrm{l}$, and tumors were allowed to form for 27 days. Tumor growth was measured every 2-3 days using calipers. The longest (L) and shortest (S) measurements were recorded, and tumor volumes were calculated as Volume $=0.5^{*}\left(\mathrm{~L} \times \mathrm{S}^{2}\right)$ and expressed as mean volume \pm SEM.

For the lung metastasis assay, cells were collected as described above and resuspended at a final concentration of $5 \times 10^{6}$ cells $/ \mathrm{ml}$ in PBS. Of this suspension, $100 \mu \mathrm{l}$ (total $5 \times 10^{5}$ cells/mouse) was injected into the tail veins of athymic nude mice (for human cell line) and C57BL/6 (for mouse cell line). After 28 days, the mice were sacrificed, and the lungs were dissected out. The number and size of metastases were assessed with minor modifications as described in previously [19]. Briefly, to determine the size and number of metastases, images from H\&Estained serial sectioning samples were taken by inverted TE300 Nikon Microscope with Spot RT Acquisition and Processing Software or Aperio ScanScope CS Slide Scanner, and the metastatic tumor size was quantified using ImageJ software or Aperio software. Numbers of metastases in lung were counted from H\&E-stained serial sectioning samples.

\section{Tissue microarray and scoring scheme}

Tks4 and Tks5 antibodies were used to stain melanoma tissue microarray samples obtained from the Cancer Diagnosis Program (CDP) of the National Cancer Institute following approval from the National Disease Research Interchange (NDRI) (http://ndriresource. org/). Antigen retrieval was performed according to the manufacturer's guidelines using Antigen Retrieval
Buffer (DAKO S1699). After incubation with Tks4 or Tks5 antibody, the sections were incubated with secondary antibody using the manufacturer's guidelines of the MACH2 Rabbit AP-Polymer (Biocare Medical) and visualized with either Vulcan Fast Red Chromogen (Biocare Medical, pink) or DAB (brown). Sections were counterstained with hematoxylin. A scoring system, developed by a trained pathologist (SI), of 0 , no staining; $1+$, low staining or staining in $<25 \%$ of tumor cells (weak positive staining); $2+$, moderate staining (positive staining); and $3+$, high staining (strong positive staining). All slides were scored blindly.

\section{Statistical analysis}

Statistical significance was determined by calculating the p-value (P) using the paired Student's $t$ test. $p<0.05$ was considered to be statistically significant. The numbers of samples (n) are indicated in each figure legend. For the immunohistochemical scoring analysis, statistical significance was determined by using the chi-squared significance test ( $p<0.05$ is significant). For 2D, 3D, and tumor growth curves, area under the curve analysis was performed on the individual growth curves using the Area Under the Curve (AUC) function in the GraphPad Prism software. Means and SEM were then calculated and Student's $t$ test was used to determine significance. AUC graphs can be found in Supplementary Figure 7.

\section{RNA isolation and qPCR analysis}

RNA was isolated from human melanoma cell lines using the RNeasy RNA isolation kit (Qiagen). cDNA was generated using the SuperScript III First-Strand Synthesis kit (Invitrogen). Absolute expression analysis was done by using standard curve for each primer sets that made by DNA plasmids containing each isoforms as template, and the gene copy number was normalized by housekeeping gene (RPLP0) to compare the expression level of each isoforms between cell lines (copy number index). Primers for each isoform of Tks5 were generated against unique regions for the alpha, beta, and short isoforms and can be found in Table 1. 


\section{ACKNOWLEDGMENTS}

We thank the personnel in the Histology, Animal and Lentivirus Production Cores at Sanford|Burnham|Prebys Medical Discovery Institute for technical assistance, the Lentiviral Service at OHSU for lentivirus production, Dr. Stefanie Kaech Petrie in the OHSU Advanced Microscopy Core for assistance with imaging, Dr. Lisa Coussens for the use of her slide scanner, Dr. Philippe Chavrier for the pHluorin-MT1-MMP construct. We thank Dr. Christine Gould for her involvement in the earlier stages of this work, and all members of the Courtneidge laboratory for discussion and input. We also thank Steven Marroulis at the Cancer Diagnosis Program (CDP) of National Cancer Institute for providing tissue microarray samples and information.

\section{CONFLICTS OF INTEREST}

The authors declare no conflicting financial interests.

\section{GRANT SUPPORT}

This work was supported by grants from the R01 CA129686 and R01 CA154002 from the NCI to SAC, and F31 CA180740 from the NCI to CA.

\section{Editorial note}

This paper has been accepted based in part on peerreview conducted by another journal and the authors' response and revisions as well as expedited peer-review in Oncotarget.

\section{REFERENCES}

1. Hanahan D and Weinberg RA. The hallmarks of cancer. Cell. 2000; 100:57-70.

2. Quail DF and Joyce JA. Microenvironmental regulation of tumor progression and metastasis. Nat Med. 2013; 19:14231437.

3. Eckert MA, Lwin TM, Chang AT, Kim J, Danis E, OhnoMachado L and Yang J. Twist1-induced invadopodia formation promotes tumor metastasis. Cancer Cell. 2011; 19:372-386.

4. Leong HS, Robertson AE, Stoletov K, Leith SJ, Chin CA, Chien AE, Hague MN, Ablack A, Carmine-Simmen K, McPherson VA, Postenka CO, Turley EA, Courtneidge SA, Chambers AF and Lewis JD. Invadopodia are required for cancer cell extravasation and are a therapeutic target for metastasis. Cell Rep. 2014; 8:1558-1570.

5. Gligorijevic B, Bergman A and Condeelis J. Multiparametric classification links tumor microenvironments with tumor cell phenotype. PLoS biology. 2014; 12:e1001995.
6. Murphy DA and Courtneidge SA. The 'ins' and 'outs' of podosomes and invadopodia: characteristics, formation and function. Nat Rev Mol Cell Biol. 2011; 12:413-426.

7. Linder S, Wiesner C and Himmel M. Degrading devices: invadosomes in proteolytic cell invasion. Annual review of cell and developmental biology. 2011;27:185-211.

8. Tarone G, Cirillo D, Giancotti FG, Comoglio PM and Marchisio PC. Rous sarcoma virus-transformed fibroblasts adhere primarily at discrete protrusions of the ventral membrane called podosomes. Exp Cell Res. 1985; 159:141157.

9. Kelly T, Mueller SC, Yeh Y and Chen WT. Invadopodia promote proteolysis of a wide variety of extracellular matrix proteins. J Cell Physiol. 1994; 158:299-308.

10. Chen WT. Proteolytic activity of specialized surface protrusions formed at rosette contact sites of transformed cells. The Journal of experimental zoology. 1989; 251:167185 .

11. Linder $\mathrm{S}$. The matrix corroded: podosomes and invadopodia in extracellular matrix degradation. Trends in cell biology. 2007; 17:107-117.

12. Gimona M, Buccione R, Courtneidge SA and Linder S. Assembly and biological role of podosomes and invadopodia. Curr Opin Cell Biol. 2008; 20:235-241.

13. Lock P, Abram CL, Gibson $T$ and Courtneidge SA. A new method for isolating tyrosine kinase substrates used to identify fish, an SH3 and PX domain-containing protein, and Src substrate. EMBO J. 1998; 17:4346-4357.

14. Seals DF, Azucena EF, Jr., Pass I, Tesfay L, Gordon R, Woodrow M, Resau JH and Courtneidge SA. The adaptor protein Tks5/Fish is required for podosome formation and function, and for the protease-driven invasion of cancer cells. Cancer Cell. 2005; 7:155-165.

15. Abram CL, Seals DF, Pass I, Salinsky D, Maurer L, Roth TM and Courtneidge SA. The adaptor protein fish associates with members of the ADAMs family and localizes to podosomes of Src-transformed cells. J Biol Chem. 2003; 278:16844-16851.

16. Stylli SS, Stacey TT, Verhagen AM, Xu SS, Pass I, Courtneidge SA and Lock P. Nck adaptor proteins link Tks5 to invadopodia actin regulation and ECM degradation. J Cell Sci. 2009; 122:2727-2740.

17. Sharma VP, Eddy R, Entenberg D, Kai M, Gertler FB and Condeelis J. Tks5 and SHIP2 regulate invadopodium maturation, but not initiation, in breast carcinoma cells. Curr Biol. 2013; 23:2079-2089.

18. Crimaldi L, Courtneidge SA and Gimona M. Tks5 recruits AFAP-110, p190RhoGAP, and cortactin for podosome formation. Exp Cell Res. 2009; 315:2581-2592.

19. Blouw B, Seals DF, Pass I, Diaz B and Courtneidge SA. A role for the podosome/invadopodia scaffold protein Tks5 in tumor growth in vivo. Eur J Cell Biol. 2008; 87:555-567.

20. Burger KL, Davis AL, Isom S, Mishra N and Seals DF. 
The podosome marker protein Tks5 regulates macrophage invasive behavior. Cytoskeleton. 2011; 68:694-711.

21. Blouw B, Patel M, Iizuka S, Abdullah C, You WK, Huang X, Li JL, Diaz B, Stallcup WB and Courtneidge SA. The invadopodia scaffold protein Tks5 is required for the growth of human breast cancer cells in vitro and in vivo. PLoS One. 2015; 10:e121003.

22. Cejudo-Martin P, Yuen A, Vlahovich N, Lock P, Courtneidge SA and Diaz B. Genetic disruption of the $\operatorname{sh} 3 p x d 2 a$ gene reveals an essential role in mouse development and the existence of a novel isoform of tks5. PLoS One. 2014; 9:e107674.

23. Murphy DA, Diaz B, Bromann PA, Tsai JH, Kawakami Y, Maurer J, Stewart RA, Izpisua-Belmonte JC and Courtneidge SA. A Src-Tks5 pathway is required for neural crest cell migration during embryonic development. PLoS One. 2011; 6:e22499.

24. Courtneidge SA, Azucena EF, Pass I, Seals DF and Tesfay L. The SRC substrate Tks5, podosomes (invadopodia), and cancer cell invasion. Cold Spring Harbor symposia on quantitative biology. 2005; 70:167-171.

25. Buschman MD, Bromann PA, Cejudo-Martin P, Wen F, Pass I and Courtneidge SA. The novel adaptor protein Tks4 (SH3PXD2B) is required for functional podosome formation. Mol Biol Cell. 2009; 20:1302-1311.

26. Iqbal Z, Cejudo-Martin P, de Brouwer A, van der Zwaag B, Ruiz-Lozano P, Scimia MC, Lindsey JD, Weinreb R, Albrecht B, Megarbane A, Alanay Y, Ben-Neriah Z, Amenduni M, Artuso R, Veltman JA, van Beusekom E, et al. Disruption of the podosome adaptor protein TKS4 (SH3PXD2B) causes the skeletal dysplasia, eye, and cardiac abnormalities of Frank-Ter Haar Syndrome. Am J Hum Genet. 2010; 86:254-261.

27. Mao M, Hedberg-Buenz A, Koehn D, John SW and Anderson MG. Anterior segment dysgenesis and earlyonset glaucoma in nee mice with mutation of Sh3pxd2b. Investigative ophthalmology \& visual science. 2011; 52:2679-2688.

28. Poincloux R, Lizarraga F and Chavrier P. Matrix invasion by tumour cells: a focus on MT1-MMP trafficking to invadopodia. J Cell Sci. 2009; 122:3015-3024.

29. Sabeh F, Shimizu-Hirota R and Weiss SJ. Proteasedependent versus -independent cancer cell invasion programs: three-dimensional amoeboid movement revisited. J Cell Biol. 2009; 185:11-19.

30. Itoh Y and Seiki M. MT1-MMP: a potent modifier of pericellular microenvironment. J Cell Physiol. 2006; 206:18.

31. Li XY, Ota I, Yana I, Sabeh F and Weiss SJ. Molecular dissection of the structural machinery underlying the tissue-invasive activity of membrane type-1 matrix metalloproteinase. Mol Biol Cell. 2008; 19:3221-3233.

32. Uekita T, Itoh Y, Yana I, Ohno H and Seiki M. Cytoplasmic tail-dependent internalization of membrane-type 1 matrix metalloproteinase is important for its invasion-promoting activity. J Cell Biol. 2001; 155:1345-1356.

33. Lehti K, Valtanen H, Wickstrom SA, Lohi J and Keski-Oja J. Regulation of membrane-type-1 matrix metalloproteinase activity by its cytoplasmic domain. J Biol Chem. 2000; 275:15006-15013.

34. Takino T, Miyamori H, Kawaguchi N, Uekita T, Seiki $\mathrm{M}$ and Sato $\mathrm{H}$. Tetraspanin CD63 promotes targeting and lysosomal proteolysis of membrane-type 1 matrix metalloproteinase. Biochem Biophys Res Commun. 2003; 304:160-166.

35. Remacle A, Murphy G and Roghi C. Membrane type I-matrix metalloproteinase (MT1-MMP) is internalised by two different pathways and is recycled to the cell surface. $\mathrm{J}$ Cell Sci. 2003; 116:3905-3916.

36. Jacob A and Prekeris R. The regulation of MMP targeting to invadopodia during cancer metastasis. Front Cell Dev Biol. $2015 ; 3: 4$.

37. Hotary KB, Allen ED, Brooks PC, Datta NS, Long MW and Weiss SJ. Membrane type I matrix metalloproteinase usurps tumor growth control imposed by the three-dimensional extracellular matrix. Cell. 2003; 114:33-45.

38. Stylli SS, I ST, Kaye AH and Lock P. Prognostic significance of Tks5 expression in gliomas. Journal of clinical neuroscience. 2012; 19:436-442.

39. Li CM, Chen G, Dayton TL, Kim-Kiselak C, Hoersch S, Whittaker CA, Bronson RT, Beer DG, Winslow MM and Jacks T. Differential Tks5 isoform expression contributes to metastatic invasion of lung adenocarcinoma. Genes \& development. 2013; 27:1557-1567.

40. Burger KL, Learman BS, Boucherle AK, Sirintrapun SJ, Isom S, Diaz B, Courtneidge SA and Seals DF. Srcdependent Tks5 phosphorylation regulates invadopodiaassociated invasion in prostate cancer cells. Prostate. 2014; 74:134-148.

41. Fidler IJ. Selection of successive tumour lines for metastasis. Nat New Biol. 1973; 242:148-149.

42. Gianni D, Diaz B, Taulet N, Fowler B, Courtneidge SA and Bokoch GM. Novel p47(phox)-related organizers regulate localized NADPH oxidase 1 (Nox1) activity. Sci Signal. 2009; 2:ra54.

43. Artym VV, Zhang Y, Seillier-Moiseiwitsch F, Yamada KM and Mueller SC. Dynamic interactions of cortactin and membrane type 1 matrix metalloproteinase at invadopodia: defining the stages of invadopodia formation and function. Cancer Res. 2006; 66:3034-3043.

44. Clark ES, Whigham AS, Yarbrough WG and Weaver AM. Cortactin is an essential regulator of matrix metalloproteinase secretion and extracellular matrix degradation in invadopodia. Cancer Res. 2007; 67:42274235 .

45. Miesenbock G, De Angelis DA and Rothman JE. Visualizing secretion and synaptic transmission with $\mathrm{pH}$ sensitive green fluorescent proteins. Nature. 1998; 394:192- 
195.

46. Lizarraga F, Poincloux R, Romao M, Montagnac G, Le Dez G, Bonne I, Rigaill G, Raposo G and Chavrier P. Diaphanous-related formins are required for invadopodia formation and invasion of breast tumor cells. Cancer Res. 2009; 69:2792-2800.

47. Egeblad $M$ and Werb $Z$. New functions for the matrix metalloproteinases in cancer progression. Nat Rev Cancer. 2002; 2:161-174.

48. Chen WT and Wang JY. Specialized surface protrusions of invasive cells, invadopodia and lamellipodia, have differential MT1-MMP, MMP-2, and TIMP-2 localization. Annals of the New York Academy of Sciences. 1999; 878:361-371.

49. Monteiro P, Rosse C, Castro-Castro A, Irondelle M, Lagoutte E, Paul-Gilloteaux P, Desnos C, Formstecher E, Darchen F, Perrais D, Gautreau A, Hertzog M and Chavrier P. Endosomal WASH and exocyst complexes control exocytosis of MT1-MMP at invadopodia. J Cell Biol. 2013; 203:1063-1079.

50. Marchesin V, Castro-Castro A, Lodillinsky C, Castagnino A, Cyrta J, Bonsang-Kitzis H, Fuhrmann L, Irondelle M, Infante E, Montagnac G, Reyal F, Vincent-Salomon A and Chavrier P. ARF6-JIP3/4 regulate endosomal tubules for MT1-MMP exocytosis in cancer invasion. J Cell Biol. $2015 ; 211: 339-358$.
51. Feldmann G, Mishra A, Hong SM, Bisht S, Strock CJ, Ball DW, Goggins M, Maitra A and Nelkin BD. Inhibiting the cyclin-dependent kinase CDK5 blocks pancreatic cancer formation and progression through the suppression of RasRal signaling. Cancer Res. 2010; 70:4460-4469.

52. Clark ES, Brown B, Whigham AS, Kochaishvili A, Yarbrough WG and Weaver AM. Aggressiveness of HNSCC tumors depends on expression levels of cortactin, a gene in the 11q13 amplicon. Oncogene. 2009; 28:431-444.

53. Chang $\mathrm{C}$ and Werb Z. The many faces of metalloproteases: cell growth, invasion, angiogenesis and metastasis. Trends in cell biology. 2001; 11:S37-43.

54. Diaz B, Yuen A, Iizuka S, Higashiyama S and Courtneidge SA. Notch increases the shedding of HB-EGF by ADAM12 to potentiate invadopodia formation in hypoxia. J Cell Biol. 2013; 201:279-292. 